\title{
Uterine Corpus Cancer pM1 TNM Finding v6
}

National Cancer Institute

\section{Source}

National Cancer Institute. Uterine Corpus Cancer pM1 TNM Finding v6. NCI Thesaurus.

Code C61358.

Uterine corpus cancer with distant metastasis, including intra-abdominal lymph nodes

other than para-aortic or inguinal lymph nodes, excluding metastasis to the vagina, pelvic serosa, or adnexa. (from AJCC 6th Ed.) 